\title{
A Self-Organized Critical Model for Evolution
}

H. Flyvbjerg, P. Bak, M. H. Jensen and K. Sneppen

\section{Abstract}

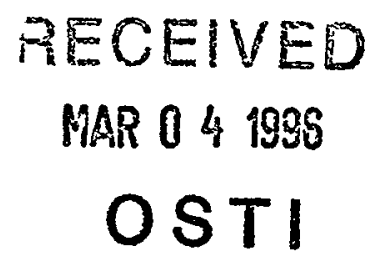

A simple mathematical model of biological macroevolution is presented. It describes an ecology of adapting, interacting species. Species evolve to maximize their individual fitness in their environment. The environment of any given species is affected by other evolving species; hence it is not constant in time. The ecology evolves to a "self-organized critical" state where periods of stasis alternate with avalanches of causally connected evolutionary changes. This characteristic intermittent behaviour of natural history, known as "punctuated equilibrium," thus finds a theoretical explanation as a selforganized critical phenomenon. In particular, large bursts of apparently simultaneous evolutionary activity require no external cause. They occur as the less frequent result of the very same dynamics that governs the more frequent small-scale evolutionary activity. Our results are compared with data from the fossil record collected by J. Sepkoski, Jr., and others.

\section{Introduction}

There is a good deal of evidence that biological evolution is not gradual, but episodic, with long periods of stasis interrupted by bursts of rapid activity. This intermittent pattern has been observed for the evolution of single species, as represented by their morphology. It has also been observed across taxa. In particular, Raup, Sepkoski, and Boyanian [1-4] have found a similar pattern for the distribution of extinction events, by studying fossil records. Gould and Eldredge [5] have coined the term punctuated equilibrium to describe the intermittent behavior of the evolution of single species; see [6] for a review and documentation of the phenomenon. We shall use the term to describe the intermittent nature of evolution in general.

Punctuated equilibrium is sometimes incorrectly presented as an explanation or a theory of the observed intermittency. Rather, it is a phenomenological principle, describing certain empirical features of the fossil record. The 
fundamental cause of evolutionary change is explained by Darwin's theory [7] which locates it to the natural selection operating by struggle among individual organisms for reproductive success. Darwin's theory may thus be thought of as the "atomic theory" for evolution. However, there is no theory deriving the consequences of Darwin's principles for macro-evolution. This is the challenge we are responding to here.

By studying the stratigraphic records of 19.897 fossil genera, Raup, Sepkoski and Boyanian found that not only do the extinction events occur in bursts within families, but different genera often show the same extinction profile. It thus appears that the evolution of different families "march to the same drummer." Some extinction events are regional [8], and the largest events are global. It has therefore been suggested that extinction events are caused by external forces, such as changing sea levels [9], worldwide climatic pulses [10], or meteorites [11]. Although this has perhaps caused some extinction, we shall nevertheless argue that punctuated equilibrium may well be the natural consequence of the dynamics of biology itself, with no need for external triggering mechanisms.

Indeed, large dynamical systems have a tendency to evolve, or selforganize, into a "critical" non-equilibrium state characterized by bursts, or avalanches, of dynamical activity of all sizes [12]. This behavior is known as self-organized criticality (SOC) and below we discuss how such behaviour may appear in an ecology driven by Darwinian evolution.

The present chapter is not the first one theorizing that the intermittency of biological evolution might be caused by self-organized critical behaviour. But theoretical investigations have been hampered by the difficulty of constructing even remotely realistic, yet tractable mathematical models. First, " "Gunctuated equilibria were observed by Bak, Chen, and Creutz [13] in the "Game of Life," a simple computer caricature of a society of living and dying individuals living on a two dimensional lattice. However it is not robust against small changes in rules, as it should be in order to represent real evolution. Later, Kauffman and Johnsen [14] in a very imaginative work studied the so-called "NKC-models" for co-evolving species evolving with periods of stasis interrupted by co-evolutionary avalanches. It was argued that the ecology as a whole was "most fit" at the critical point. However, as these models where driven they do not self-organize: some external tuning of the system, "divine intervention," was needed to obtain critical behaviour $[15,16]$. Finally, intermittent bursts indicating evolution to a critical state have been observed in computer simulations of reproductive organisms, first by Ray [17], and very recently by Adami [18].

This chapter is organized in the following way. In Section 2 we present our model. In Sections 3 and 4 we discuss analytically tractable versions of it. Readers less keen of mathematical details should skip these sections. In section 5 we discuss a version that is easily simulated. In section 6 we discuss pertinent data from the fossil record in the light of our results. In 


\section{DISCLAIMER}

This report was prepared as an account of work sponsored by an agency of the United States Government. Neither the United States. Government nor any agency thereof, nor any of their employees, makes any warranty, express or implied, or assumes any legal liability or responsibility for the accuracy, completeness, or usefulness of any information, apparatus, product, or process disclosed, or represents that its use would not infringe privately owned rights. Reference herein to any specific commercial product, process, or service by trade name, trademark, manufacturet, or otherwise does not necessarily constitute or imply its endorsement, recommendation, or favoring by the United States Government or any agency thereof. The views and opinions of authors expressed herein do not necessarily state or reflect those of the United States Government or any agency thereof. 

section 7 we discuss the characteristics of life in an ecology that has reached the self-organized critical state predicted by all version of the model. Section 8 contains the discussion and conclusions.

\section{Modelling}

Our investigation starts at the level of species. We consider the microevolution acting up to this level as decoupled from the macroevolution that we wish to understand. This decoupling, of course, is not a claim of falseness or irrelevance of microscopic mechanisms. It is an assumption that divides the problem into more manageable parts.

The basic picture that we have in mind is the evolutionary fitness landscape envisioned by Wright in his seminal work, the shifting balance theory of evolution, reviewed in [20]. The properties of a population are modified by means of mutation and differential selection towards higher fitness. Random mutations allow individuals to cross barriers of lower fitness and move to other maxima, and initiate a population at or near this new maximum.

For simplicity, we define a species as a group of individuals in the vicinity of the same fitness peak. The basic evolutionary step in our theory is the transformation of one species to a similar, more fit, species. We call this step a "mutation" of the species, following Gould [6]. The detailed mechanisms making this step possible is not of our concern: we refer to the work of others for its motivation. For instance, the diffusion of a species from one state to another has been described by Lande [21], and by Newman et al. [22]. The mechanism is mutation and differential selection of the fitter variant, causing the whole population to evolve to this variant. Figure 1 shows how this step may take place in a laboratory experiment. The figure shows the mean scutellar bristle number in female Drosophila Melanogaster according to MacBean et al. [23], as simplified by Parsons [24]. The number jumps to a "fitter" value in response to selective pressure.

In principle, the "fitness" landscape for a given species can be expressed as a function of its genetic code $[25,14]$, and also of the genes of certain other species that it depends on. However, we ascend another step in the level of abstraction: we assume that the fitness landscape is sufficiently rough that escape of a species from one local fitness maximum to another results in the replacement of one effective barrier towards further evolution with another effective barrier. We further assume that these subsequent barriers are uncorrelated. With this we have done away with many details of any specific model and obtained generality and mathematical simplicity in return. Our choice between what to leave out, and what to leave in, in the simplified model is an expression of what we consider quintessential mechanisms of evolution, mechanisms so fundamental that they should also characterize life on Mars, if it existed, and Ray's computer life in the Tierra environment [17]. 


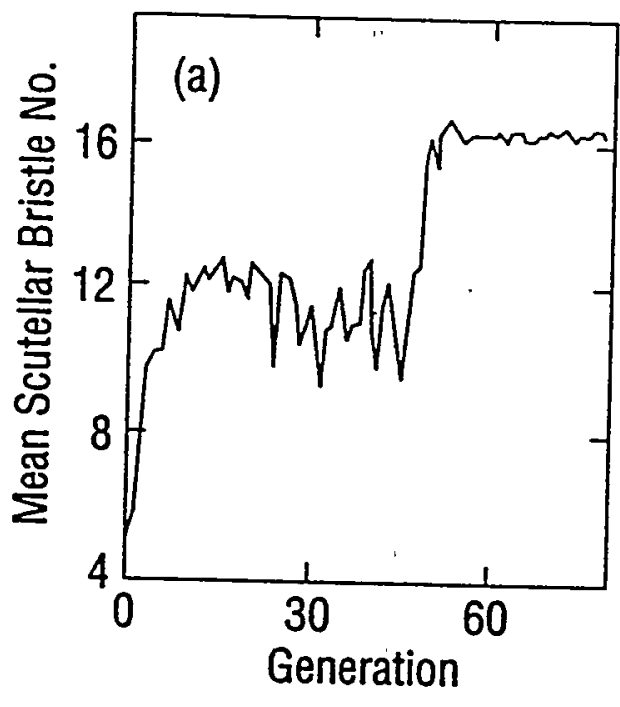

Fig. 1. "Punctuation" in mean scutellar bristle number in female Drosophila Melanogaster as observed in the laboratory by MacBean et al. [23].

The probability of jumping from one state to a better one is $p=e^{-B / T}$, where $B$ is a random number expressing the barrier, i.e. number of single base pair mutations, separating the two states, and $T$ is an effective mutation parameter defining the timescale of mutations. Notice that although the typical time between jumps from one state to another is large, i.e. $1 / p$ small, the jump itself is very fast [21]. A species with a high fitness is unlikely to evolve to even higher fitnesses, so its barrier $B$ is high, whereas a species with low fitness have an easy time doing this. Thus, the barrier of stability $B$ can
be thought of as a measure of fitness.

All of the above describes the evolution of a single species in a given landscape. However, as pointed out by Kauffman and Johnsen [14], the fitness landscape experienced by one species depends on other species in the ecology: it is a "rubber" landscape, changing with the physical properties of other species and therefore with their genes. The interacting species can for instance be consecutive links in a food chain. As the fitness of one species improves, the fitness of its neighbors is affected, typically making some of them likely
to evolve. Thus species co-evolve.

We model this by assuming that the fitnesses of these neighbors assume new values, either directly as a consequence of the different environment, or because they quickly move to a new local fitness maximum, with a new, by assumption random, barrier value $B$ towards further evolution. But in the course of doing so they may have induced yet other species to a fast evolution to new local fitness maxima with new random barrier values towards further evolution. We assume that the interactions between species are sufficiently weak or dilute to allow this primary chain reaction of co-evolution to die out fast. Thus, in such a brief chain reaction only a finite number of species, say $K$ on the average, are affected and we choose a time-scale in our model in which the chain reaction is represented by a single step. 


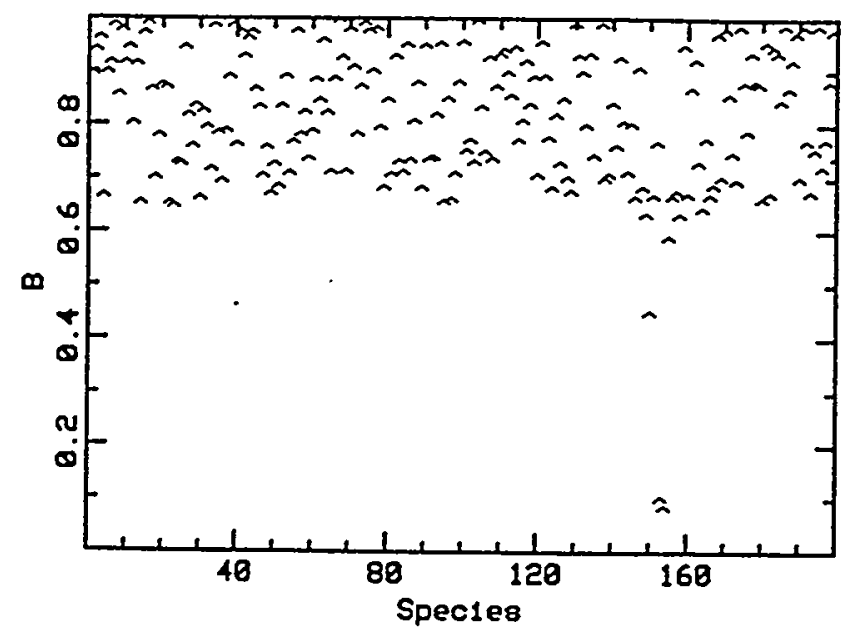

Fig. 2. Illustration of the "food-chain" version of our model. A snapshot of the barrier values for the different species are shown. Most barriers are above the critical value $B_{c}=0.667$. In the next step, the species with the lowest barrier, here number $i=790$, will mutate.

For the sole purpose of simplifying the bookkeeping of the network of interactions among species, let us assume first that the network forms a simple chain. We may then think of species as placed on a straight line, each species interacting with its two nearest neighbors on the line (Figure 2). Initially we choose random values $B$ between 0 and 1 for the barriers towards further evolution experienced by the species. The choice of initial condition does not affect the outcome of evolution in this model. In the limit where the mutation parameter $T$ is low, the first species to mutate is always the one with the lowest barrier. I.e. the first species to succumb is the least fit, as envisioned by Darwin.

Thus, the dynamics is as follows [26]: The species $i$ with the lowest barrier is assigned a new random value of $B$ between 0 and 1 . Its two neighbors are also to be assigned new random numbers. Therefore, even if any of these neighbors had a high fitness, this property is likely to be lost. In the next step, the species which now has the lowest fitness mutates, affecting the fitness of its neighbors, and so on.

The actual rate of evolution varies enormously with the value of the minimum barrier representing the next species likely to mutate spontaneously. As the system evolves it turns out that no species with barrier values above a certain self-organized threshold, $B_{c}$ (equal to 0.667 in the case considered here), will ever mutate spontaneously: They evolve only when their environment has changed sufficiently to lower their barriers. No high barriers towards evolution are ever transgressed. No highly improbable events are required in evolution. Instead, the improbable is made probable by changes in the environment; the "rubber" landscape of a species co-evolving with other species is modified by them to allow successive small, but rapid, evolutionary steps. 


\section{Random Neighbor and Mean Field Model}

Here, for mathematical convenience, we select the $K-1$ interacting species at random among the $N$ species in the ecology. This Random Neighbor Model is a first step towards a solvable mean field theory [27]. We also assume this randomness to be "annealed", i.e. the next time the same species triggers $K-1$ other species to evolve, they are chosen at random anew. A mean field theory can be constructed by neglecting correlations between barrier values. Then the $i$ th smallest barrier value, call it $x_{i}$, is distributed as the $i$ th smallest number out of $N$ drawn from the distribution $p(x, t)$ of all barrier values in the ecology. If we let $p_{i}$ denote the distribution for $x_{i}$ then our mean field approximation is the assumption that

$$
p_{i}(x)=\frac{N}{(i-1)[(N-i)]} P^{i-1}(x) p(x) Q^{N-i}(x),
$$

where we have introduced

$$
\begin{aligned}
& P(x)=\int_{0}^{x} d x^{\prime} p\left(x^{\prime}\right) . \\
& Q(x)=\int_{x}^{1} d x^{\prime} p\left(x^{\prime}\right)
\end{aligned}
$$

Normalization of $p$ gives

$$
\int_{0}^{1} d x^{\prime} p\left(x^{\prime}\right)=P(x)+Q(x)=1 \forall x
$$

We can easily write down the evolution equation for $p(x, t)$,

$$
\begin{aligned}
p(x, t+1)= & p(x, t)-\frac{1}{N} p_{1}(x, t) \\
& -\frac{K-1}{N-1}\left(p(x, t)-\frac{1}{N} p_{1}(x, t)\right)+\frac{K}{N},
\end{aligned}
$$

where Eq. (1) gives the distribution for the smallest barrier,

$$
p_{1}(x)=N p(x) Q^{N-1}(x),
$$

whose removal from the set of $N$ barrier values is represented by the second term on the right-hand side of Eq. (5). The third term on the right-hand side of Eq. (5) represents the removal of $K-1$ of the $N-1$ barrier values remaining after the smallest has been removed from the set of $N$ values. These $K-1$ values can be any of the $N-1$ values remaining, hence are distributed as these, i.e. as $\left(N p(x, t)-p_{1}(x, t)\right) /(N-1)$. The last term on the right-hand side of Eq. (5) represents the addition of $K$ new equi-distributed barrier values, replacing the $K$ values that were removed with the preceeding terms. Notice that probability is conserved by Eq. (5). 
Our mean field dynamics is an approximation to the master equation for the Markov process of the random neighbor model, both having one unique attractive fixed point. At this fixed point Eq. (5) is an integral equation fo $p(x)$, or, equivalently, an ordinary differential equation for $Q(x)$. It is solved by the positive root $Q(x)$ of the polynomial equation

$$
\begin{aligned}
(N-K) Q^{N}(x) & +N(K-1) Q(x) \\
& +(N-1) K(x-1)=0 .
\end{aligned}
$$

In the limit where $N \gg K>1$, the first term in this equation is small relatively to the second term for such values of $x$ where $Q(x)$ is less than 1 by more than $\mathcal{O}(1 / N)$. Consequently we have

$$
\begin{aligned}
Q(x) & =\frac{(N-1) K}{N(K-1)}(1-x)-\frac{N-K}{N(K-1)} Q^{N}(x) \\
& \simeq \frac{K}{K-1}(1-x) \text { for } x-1 / K \gg \mathcal{O}(1 / N) .
\end{aligned}
$$

Conversely, where $Q(x) \simeq 1$ we have

$$
\begin{aligned}
Q(x) & =\left(\frac{(N-1) K}{N-K}(1-x)-\frac{N(K-1)}{N-K} Q(x)\right)^{1 / N} \\
& \simeq(1-K x)^{1 / N} \text { for } 1 / K-x \gg \mathcal{O}(1 / N) .
\end{aligned}
$$

Using $p(x)=-\frac{d}{d x} Q(x)$, we have

$$
\begin{aligned}
& p(x) \simeq \frac{K}{N} \text { for } 1 / K-x \gg \mathcal{O}(1 / N) \\
& p(x) \simeq \frac{K}{K-1} \text { for } x-1 / K \gg \mathcal{O}(1 / N) .
\end{aligned}
$$

The exact solution of Eq. (7) is easily obtained numerically by iteration of Eqs. (8) and (9) for $x>1 / K$ and $x<1 / K$, respectively. It is shown in Figure $3 \mathrm{a}$ together with the resulting distribution of the smallest barrier, $p_{1}(x)$, both as dashed lines. The random neighbor model is easily simulated and its equilibrium distributions $p(x)$ and $p_{1}(x)$ are shown in Fig. 3a as full lines.

In the limit $N \rightarrow \infty$ we see that $p(x)$ has a discontinuity at $x=1 / K$; it vanishes below this threshold and is constant above it. It is easy to understand this result in approximate terms: Suppose $p(x) \simeq K / N$ for $0 \leq x \leq 1 / K$ and $p(x) \simeq K /(K-1)$ for $0 \leq 1 / K \leq x$. Then the smallest of $N$ barrier values distributed according to $p$ will be equi-distributed below the threshold value $1 / K$, and the other $N-1$ will be larger than $1 / K$, typically. Thus, when the smallest barrier value is removed none is left below threshold. Consequently, the $K-1$ additional barrier values which are randomly selected and removed, must be taken from above the threshold, hence are equi-distributed 

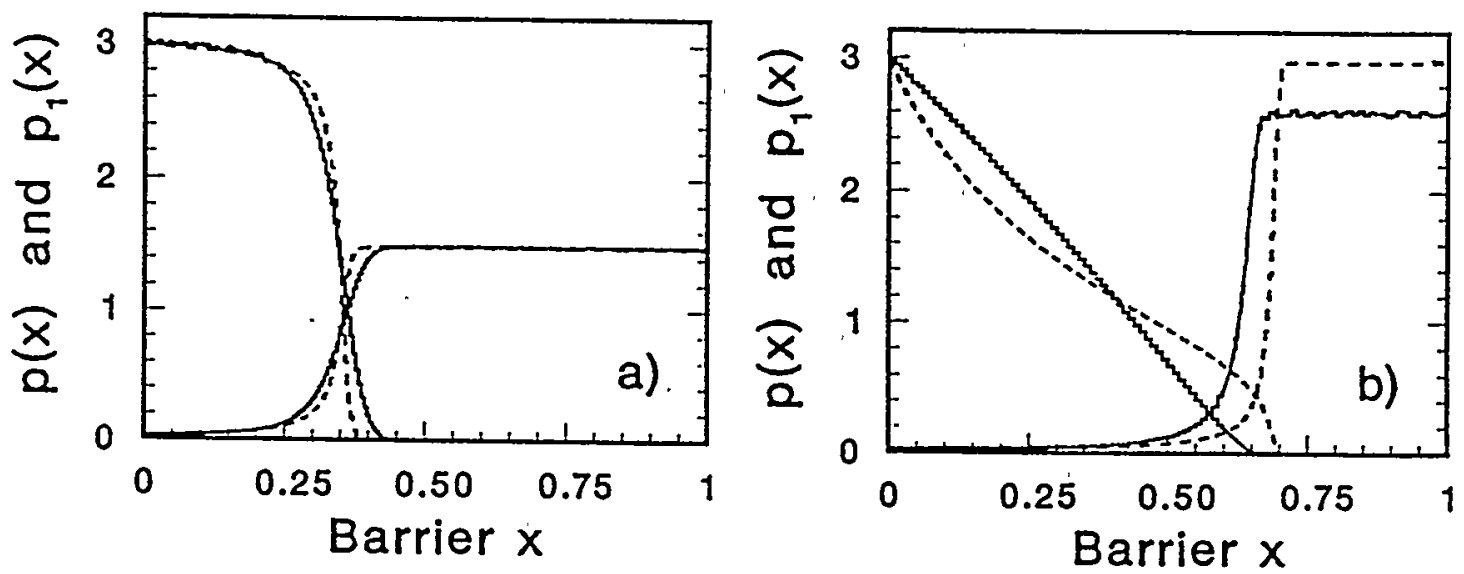

Fig. 3. (a) Equilibrium distribution of barrier values $p(x)$ and distribution of smallest barrier value $p_{1}(x)$ for simulated random neighbor model (full curves) and corresponding mean field theory (dashed curves). (b) Same distribution for $1 \mathrm{~d}$ model (full curves) and mean field theory removing two lowest barrier values (dashed curves). All cases have $K=3$ and $N=100$.

by assumption about $p$. When we replace these $K$ barrier values with $K$ equi-distributed values, one of these typically falls below the threshold and the other $K-1$ above. Since all of them are equi-distributed, $p$ is left unchanged, as it should be.

This explanation points to another aspect of the asymptotic dynamics: If we trace in time which species trigger the bursts of evolutionary activity, then it is usually one of the species participating in recent activity. So at any given late time, the very species which acquired their current properties most recently are also the ones most apt to change them again. Thus, according to our model, the cockroach, which is much older than the human race, will resemble itself long after humans, as we know them, have disappeared.

\section{Avalanches}

In order to express the causal connections between bursts of evolutionary activity, we define an avalanche as a causally connected sequence of activity associated with barrier values below the self-organized treshold $1 / K$. Suppose that at some time all barrier values are above the threshold value. Then the next burst will, on the average, result in one barrier value below threshold, which for its part will result in another barrier value below threshold, etc. The number of barriers below threshold remains constant equal to one, on the average. The actual number of barriers below threshold fluctuates and may become zero again, terminating the avalanche.

A more realistic value for the average number of barrier values below threshold can be obtained from our mean field approximation. It gives 


$$
\begin{aligned}
N P(1 / K)= & \ln N-\ln \ln N-\ln (K-1) \\
& +\mathcal{O}(\ln \ln N / \ln N)+\mathcal{O}(1 / \ln N)+\mathcal{O}(\ln N / N)
\end{aligned}
$$

where $P(1 / K)=1-Q(1 / K)$, and $Q(1 / K)$ is the solution to Eq. (7) with $x=1 / K$. With an average of $N P(1 / K)$ barrier values below threshold, the fluctuation in this number needed to terminate an avalanche becomes increasingly rare with increasing $N$. Thus the sizes of avalanches, defined as the number of bursts they contain, grow with $N$, to diverge as $N \rightarrow \infty$.

In the limit $N \rightarrow \infty$, an avalanche defined as above can be identified with a critical branching processes with branching ratio $K$ [29]. This is done by identifying each burst with a node, and each of $K$ new barrier values resulting from a burst with either a branch rooted in that node (if the barrier value is less than the threshold value), or with a leaf rooted in the same node (if the barrier value is above threshold.) The limit $N \rightarrow \infty$ is necessary to obtain the tree structure. This identification tells us that avalanches come in all sizes $s$, and the larger ones are distributed according to a power-law with mean field exponent,

$$
D(s) \propto s^{-3 / 2},
$$

showing that there is no average size to avalanches. The avalanches are critical, because the branching process is. Since the medium through which these avalanches propagate - the set of $N$ barrier values-is transformed by the avalanches and driven by them to the unique asymptotic fixed point distribution that makes the avalanches critical, our model for biological evolution is a self-organized critical dynamical system.

By analytical means one can obtain a number of exact results for the Random Neighbor Model which was described here in the mean field approximation. The mean field description is sufficient for our purposes here, i.e. for demonstrating qualitative features shared by the Random Neighbor Model and finite dimensional models. Readers interested in exact results for the Random Neighbor Model are referred to [30].

เry

ity

ose

ihe

ld,

tc.

on

nd

\section{One-dimensional Model}

So far, we have seen criticality only in the mean field approximation. Now let us study a finite dimensional case. We have simulated the dynamics of the one-dimensional ecology and measured a number of its properties in the equilibrium state. Figure $3 \mathrm{~b}$ shows the distribution of barriers, $p(x)$, and the distribution for the lowes barrier value, $p_{1}(x)$, as full curves. Both are for $K=3, \mathrm{~N}=100$. They do not resemble the random neighbor and mean field results for $K=3, N=100$, shown in Fig. 1a. The dashed lines in Fig. 1b show results from a different mean field model, obtained also with $K=3$ and $N=100$, but by replacing the two smallest barrier values plus one 
randomly selected value with random numbers in each time step. It is easy to understand why this latter algorithm gives results much closer to the 1d results: low barrier values are clustered in one dimension, so the replacement of the smallest barrier value together with the values on its nearest neighbor sites amounts to replacing the lowest value plus 0-2 other low values. Actually, some of the difference between the mean field and 1d results shown in Fig.1b is due to finite- $N$ effects being more pronounced in the $1 \mathrm{~d}$ results. For example the value of $p(x)$ for $x>0.7$. It will approach 3 as $N \rightarrow \infty$, while the mean field value for $p(x)$ is already very close to 3 for $x>0.7$.

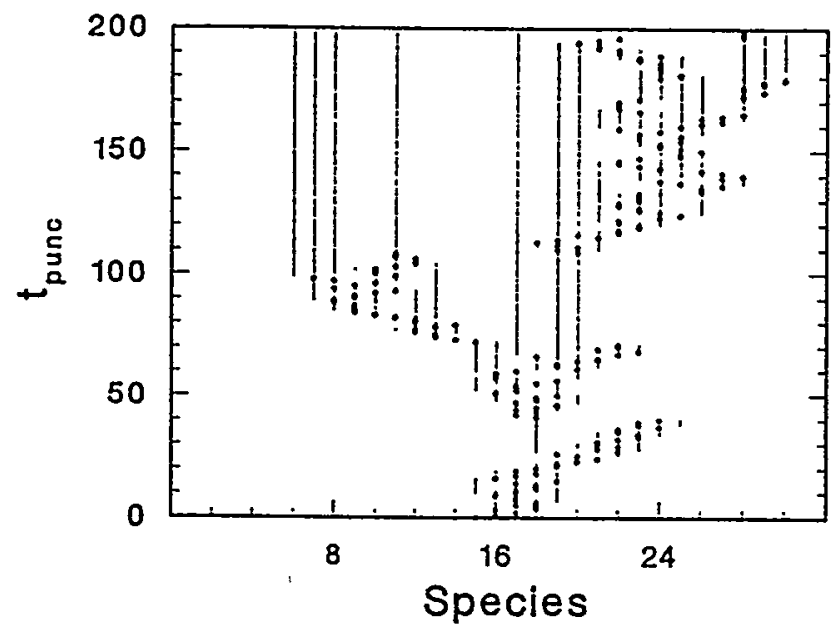

Fig. 4. Space-time map of an avalanche in the self-organized critical state. At any time the site with minimum barrier value is shown as a large dot. Sites with barrier values below the threshold value 0.67 are shown with a small dot. The activity is seen to always return to sites below threshold.

Figure 4 shows a space-time map of those sites on which species change barrier values in the time interval covered. Whenever the lowest barrier value is found among those $K=3$ last renewed, the site of lowest barrier value performs a random walk, because those 3 sites have equal probability for being the one with smallest barrier value. The figure shows that this is what happens most frequently. When the site of lowest barrier value moves by more than one lattice spacing (jumps), it most frequently backtracks by two lattice spacings to a site that was updated in the next-to-last time step. But longer jumps occur, too; actually jumps of any length occur, as indeed they must in order to be consistent with results below. These jumps always take the walker back to a site that was updated recently, the longer jumps typically to a less recently updated site. In popular terms, the site of lowest barrier value is a "jumpy random walker with a repetitious compulsion".

Figure 5 shows three aspects of this repetitious random walk which differ from a truely random walk: The root mean square of the distance traveled vs time and the number of different lattice sites visited as a function of time both 


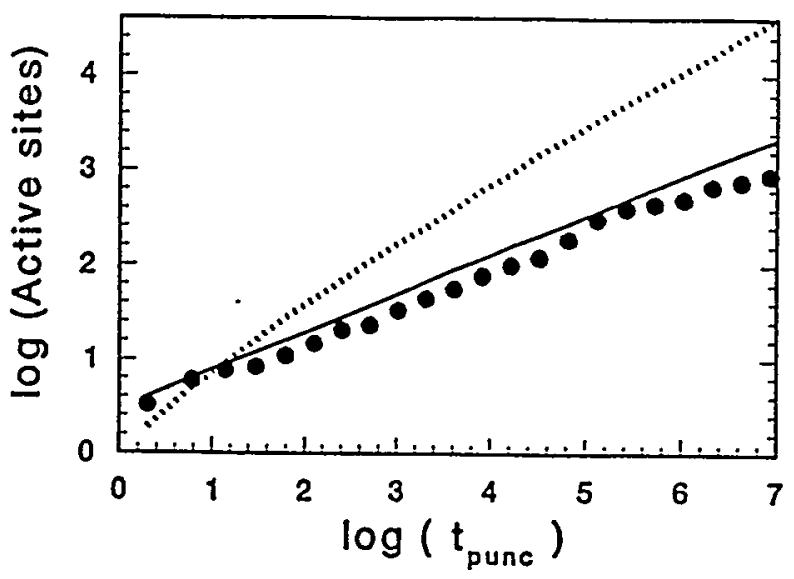

Fig. 5. Large dots: Displacement of the activity Large dots: Displacement of the activity as a function of time, starting at an arbitrary time in the critical state. This quantity grows as $t^{0.43}[28]$.

grow with exponent 0.40 , in contrast to the exponent $1 / 2$ obtained for the random walker. The maximum number of updates of any state as a function of time appears to grow as $t^{0.6}$. This exponent may have a simple explanation in terms of the others: the total number of updates is proportional to time, and the number of different sites visited to $t^{0.4}$. So the number of visits to a given site (in particular to the one most often visited) should grow with exponent $1-0.4=0.6$. For the random walker this relationship reads $1-1 / 2=1 / 2$ for the number of visits to any site, for instance the origin of the walk.

The biological implication of this correlated spreading of evolutionary activity is that species that evolved recently are also most likely to change again. cf. humans vs cockroaches above. The actual values of the exponents, here 0.4 and 0.6 , depend on the dimension, here chosen equal to one. If our model has an upper critical dimension above which mean field theory is exact, and this dimension is a small integer, the mean field version of our model is probably the most relevant one to use in an analysis of historical biological data.

\section{The Theory vs. the Fossil Record}

Figure 6a shows a time series for the relative number of species becoming extinct in consecutive intervals of approximately 5 million years, as presented by J. Sepkoski, Jr. [4]. Note the intermittent behavior with a few large peaks representing mass extinction events and many small peaks and valleys representing periods with smaller relative numbers of extinctions. Figure $6 \mathrm{~b}$ shows similar results from a numerical simulation of our model.

Figure 7a shows a histogram of essentially the same data as those presented by Raup [2]. The histogram shows the number of genera becoming extinct in each of 106 time intervals covering approximately 5 million years 

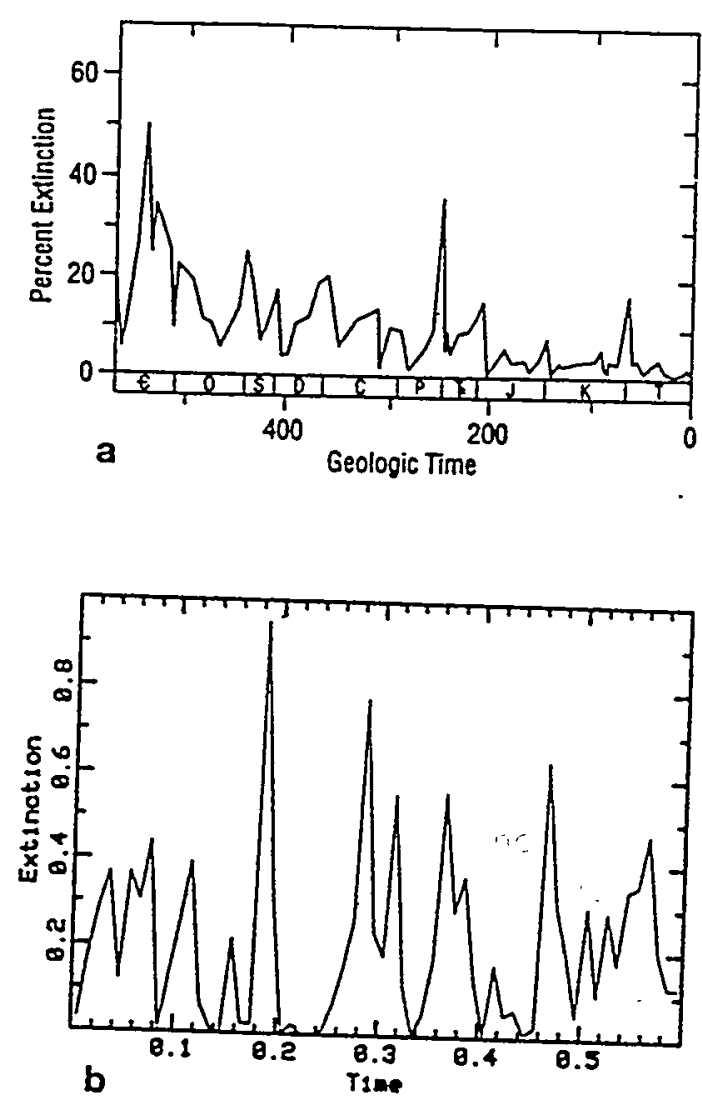

Fig. 6. 'Temporal evolution' of extinctions recorded over the last 600 million years, as given by $J$. Sepkoski (4). The ordinate shows estimates of the percentage of species that went extinct within intervals of 5 million years. (b) Temporal evolution of the "mutation" activity of species recorded in a 1-dimensional model ecology with 200 "species" and a mutation rate parameter $T=0.01$.

each. The distribution is highly skewed, with 52 of the intervals having less than 10 percent extinction, and a few large extinction events with up to 60 percent extinction. The histogram varies fairly smoothly between the periods with small activity and those with large events. This smoothness suggests that a common mechanism is responsible for all events.

Figure $7 \mathrm{~b}$ shows a similar histogram for a subset of families, including 2316 marine animal families. Again, a smooth variation is observed. Of course, because of the small number of periods there are very large statistical variations, in particular for the few large events. Actually, these large events are so few that they each have names.

Figures $7 \mathrm{c}$ shows further results from numerical simulations of our model. The quantity measured in these simulations is the number of "mutations" of species occurring, rather than extinction events. The time series shows the characteristic behavior with long periods of little activity, interrupted by peaks of large evolutionary activity. The histogram shows the smooth transition from the many periods with little activity to the few large catastrophic events. Mathematically, the histogram of events of size $s$ follows a power law, $N(s)=s^{-\tau}$, with the exponent $\tau$ ranging from 1.1 to 1.5 depending on a certain feature of the simulated, theoretical ecology (its so called "imbedding dimension"). The histogram shown is for randomly connected species (infinite imbedding dimension). Our theory confines the value of the activity exponent 

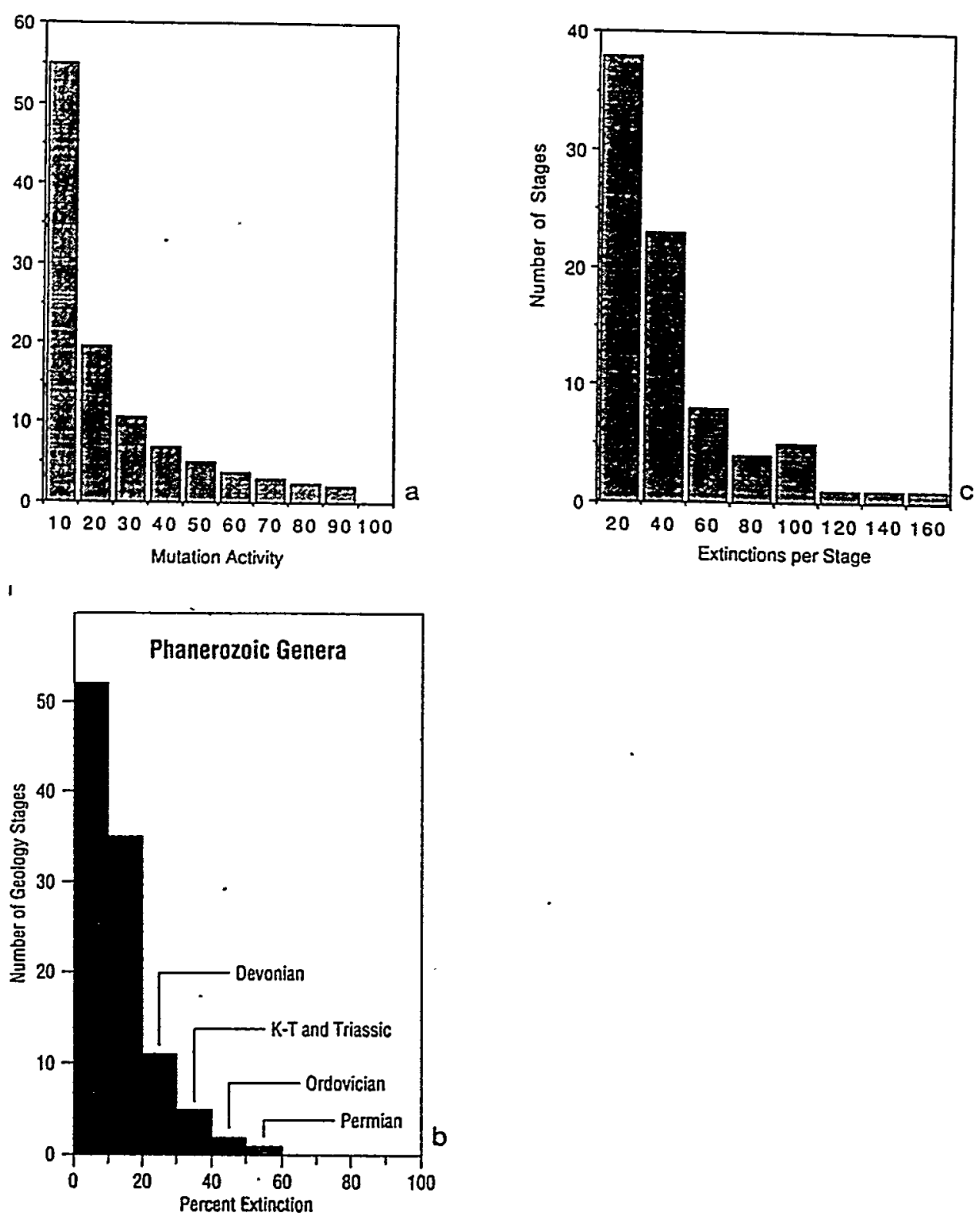

Fig. 7. (a) Histogram of extinction events from Fig. 6a, as shown by Raup. The extinctions are binned in 106 intervals of approximately 5 million years each. (b) Distribution of extinction events based on recorded time of extinction of 2316 marine animal families. (c) Histogram of mutation activity as predicted by the random neighbour version of our model.

$\tau$ to this very narrow range, whereas actual data for extinction events appear slightly steeper than an ecology of randomly connected species allows for. But the data is too scanty to allow for a real quantitative test of the theory.

Figure 8a shows the time series for a different type of data, namely the variation of the morphology of a single species. The figure shows the increase in thoractic width of the Antarctic radiolarian Pseudocubus vema during 2.5 million years according to Kellogg [19]. The figure shows punctuated equilib- 

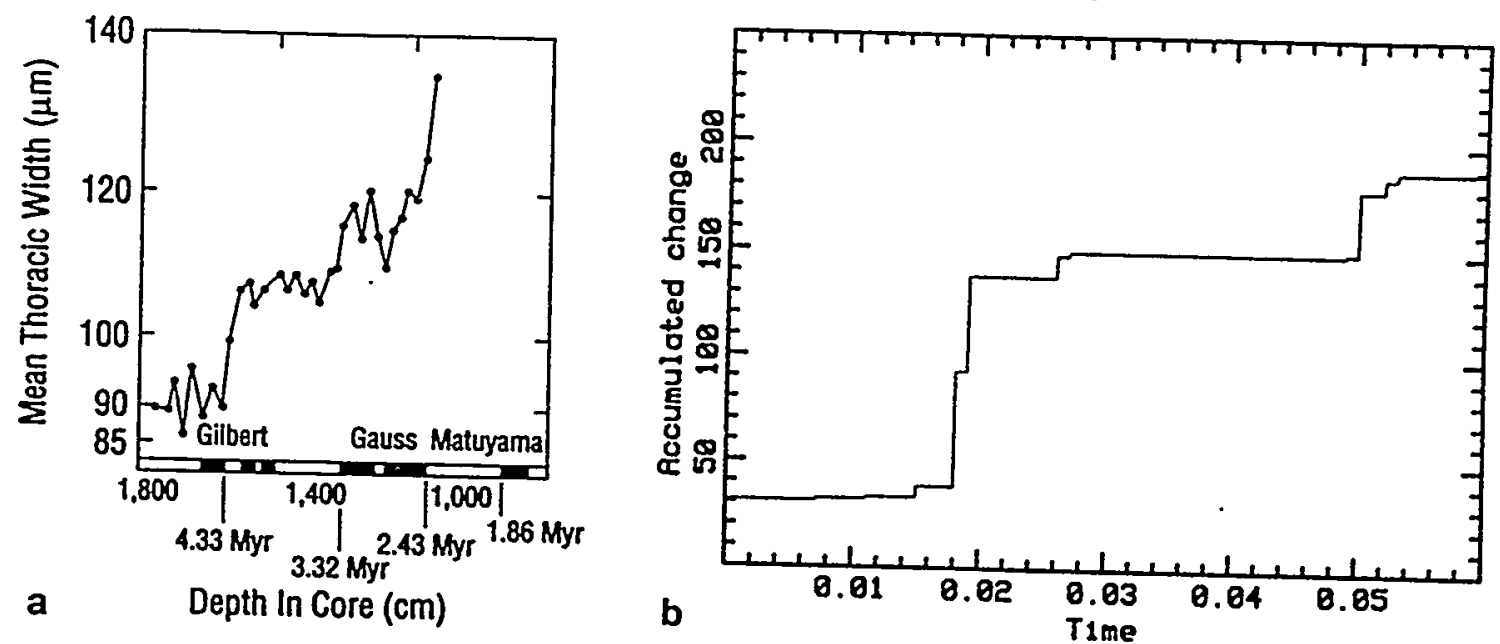

Fig. 8. (a) Time series for the variation of the morphology of a single species. The figure shows the increase in thoractic width of the Antarctic radiolarian Pseudocubus vema during 2.5 Myr according to Kellogg [16]. (b) Model prediction for time series for change of single species morphology, estimated as its accumulated mutational activity.

ria with a series of three plateaux, i.e. periods of stasis, separated by periods of rapid change. Figure $8 \mathrm{~b}$ shows similar behavior from our simulations. Again, the distribution of jumps can be shown as a histogram, with power law distribution of bursts.

In our theory, the evolution of single species is coordinated. Figure 9 shows the time series of global extinctions together with the evolution of a single species. The bursts of rapid variation take place during periods of large biological activity.

The most interesting feature of evolution is, perhaps, the existence of periods of stasis. During such periods species in an ecology seem to be in balance. Figure 10a shows a histogram of the lifetimes of species based on data on 17505 genera tabulated by Sepkoski, as presented by Raup [1]. The distribution varies smoothly from very many species with short life span, to few species with long life span, up to several hundred million years. The number of species $N$, with a life-time $t$ can be fitted quite well to a power law, $N(t) \propto 1 / t^{\alpha}$ with $\alpha \approx 2$. Figures. 10bc shows the results of our simulation from the one-dimensional version of the model. Our measure of lifetime is the interval between successful mutations of a given species. A mutation event can be thought of as an extinction event followed by the replacement by another species. The theoretical distribution of lifetimes from our model is also a power law, however with an exponent $\alpha \approx 1$ for small times, and much steeper when measured over times that are comparable to the times between the largest events. 


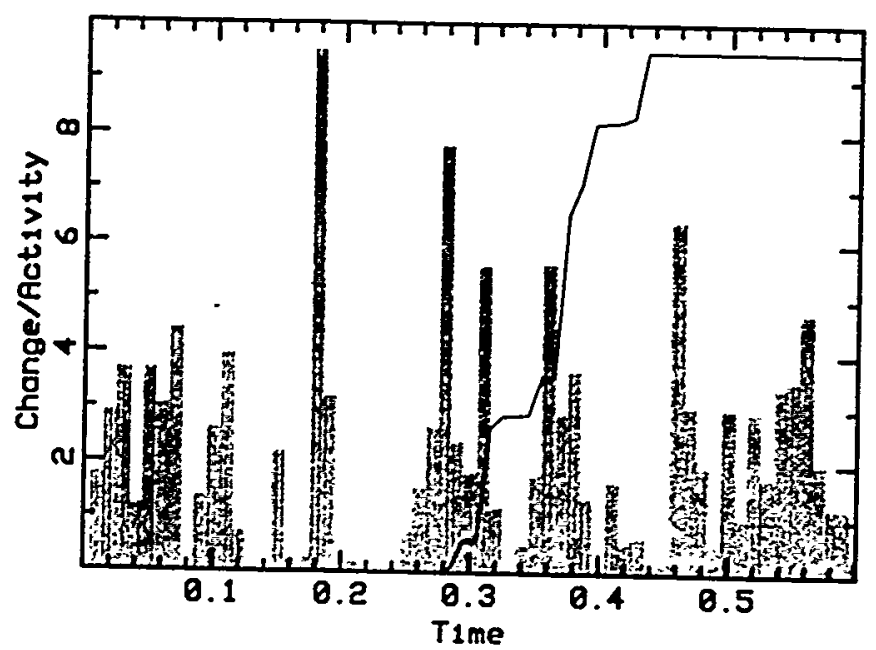

Fig, 9. Model predicted time series of global extinctions together with the evolution of a single, randomly chosen, species. The bursts of rapid variation takes place
during periods of large biological activity.

\section{Life in the Self-Organized Critical State}

As evolution proceeds, the ecology organizes itself into a state where further evolution takes place as avalanches of hectic co-evolutionary activity, or "punctuations" in the language of Gould and Eldredge. An avalanches may be defined rigorously as the total activity during a period where at least one species has a barrier below the critical threshold $B_{c}$. During an avalanche, there are several species with relatively low fitness. Between avalanches, in periods of stasis, the fitnesses of all species are above a threshold, so nature appears to be in balance.

The magnitude, or size, of an avalanche, or punctuation, is defined as the total number of successful evolutionary moves constituting the avalanche. If one plots a histogram of the number of avalanches of a given size $s$, one finds that the size distribution is a power law, $N(s)=s^{-1.1}$ [28]. This power law distribution indicates that the system is in a critical state, with a terminology borrowed from theoretical physics. Avalanches of all sizes occur, including large catastrophic one. This is in contrast to normal, or Gaussian distributions which have exponentially small tails that effectively prohibit large events.

So the catastrophic events occur due to a collective behavior of the interacting species, each of which is trying to gain maximum fitness in its landscapethe replacement by another species. The model says nothing about the actual nature of the interaction leading to extinction of a specific specie. It could be the result of falling prey to other species, starvation from change in habitat or competition from other species, epidemics, in short, the usual fates of the weak.

The important point is that in this model there is no need for external causes, such as climatic changes, to explain the observed pattern of extinction 

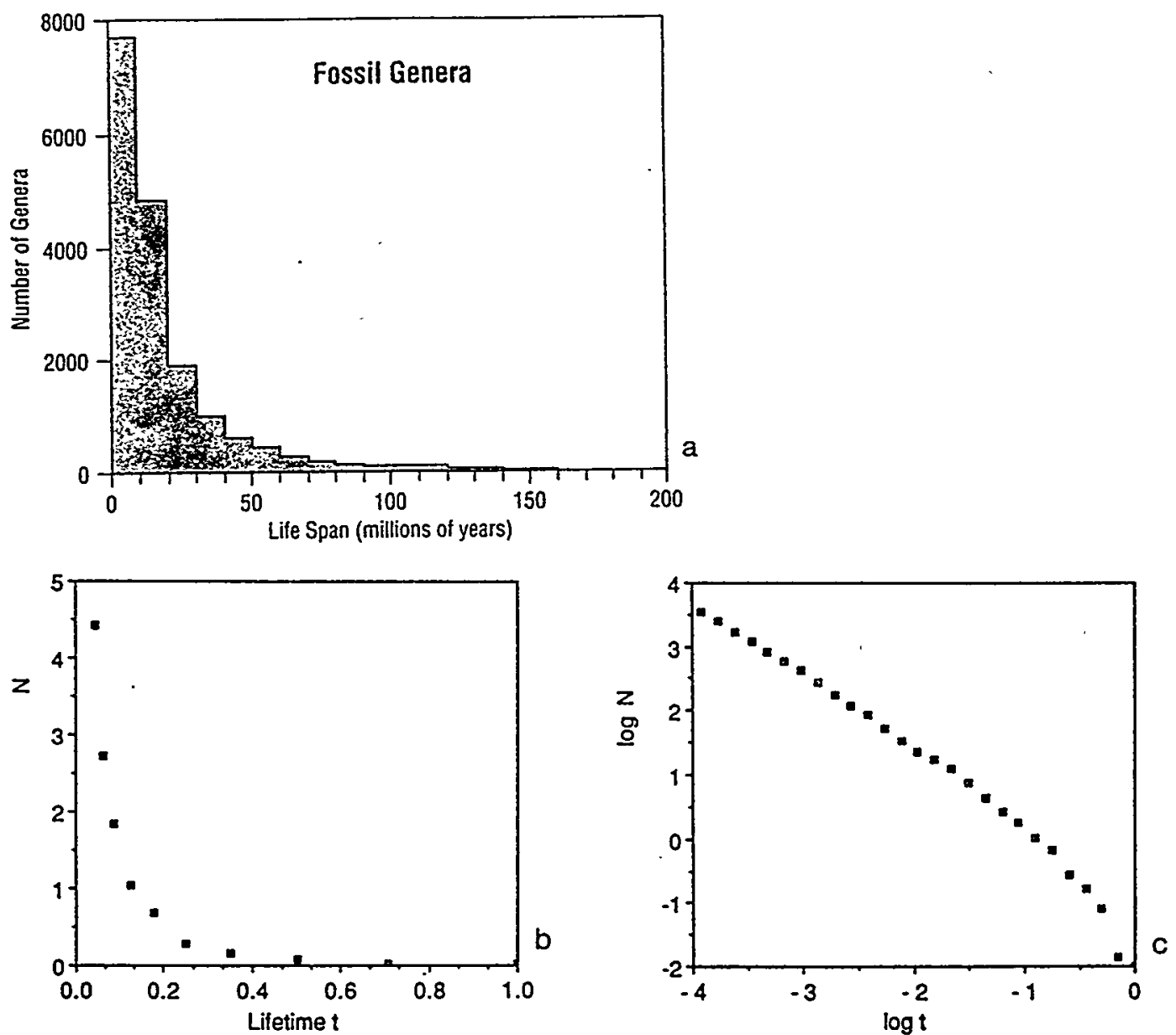

Fig. 10. (a) Life time distribution for species as recorded by Sepkoski et al. [1-4]. The distribution can be well fitted by a power law $N(t) \propto 1 / t^{2}$. (b) Distribution of life times for the 1-d model for a mutation parameter $T=0.001$. Time is measured in units of $\exp \left(B_{c} / T\right)$. (c) $\log -\log$ plot of the same distribution. For small times the distribution is $\propto 1 / t$.

events, even when these cut across functional, physiological and ecological lines. In particular, no external cataclysmic impact is necessary in order to generate large events in evolution. The late Permian extinction event with an estimate of up to $96 \%$ species going extinct [1], or the late Cretaceous event opening for the early Tertiary evolutionary radiation of mammals, may thus have been endogenous events in evolution.

Returning to our artificial lattice arrangement of the network of interactions between species, the exponent of the power law depends on the dimension of the lattice [26-28]. On a two dimensional lattice the exponent is $1.27 . .$. , in higher dimensions it is yet higher, but the exponent does not exceed $3 / 2$, actually has that value for all lattice dimensions larger than 4 . If the network of interactions is chosen to be random-a choice that presumably resembles reality more than any lattice network - the exponent can be calculated analytically to be exactly $3 / 2$ [27], and a number of other proper- 
ties can be derived analytically as well [29]. Figure 7c shows a histogram of the size of avalanches as calculated here.

Since the minimum barrier value fluctuates much, the actual time scale represented by a single mutation in our computer simulation varies enormously. In order to represent the evolution on a real time scale, simulations have been performed at a low value of the mutation parameter, $T=0.01$. At each time step, a given species with barrier $B_{i}$ mutates with the probability $p=\exp \left(-B_{i} / T\right)$. When a species mutates, we assign both it and its two neighbours new random barrier values $B$ between 0 and 1 . Figure 11 shows a space-time plot of the activity. The horizontal axes is the "species axis," and the time where a given species mutates is shown as a black dot. The avalanches are shown as connected black areas. On the time resolution of the plot, the avalanches appear as almost horizontal lines.

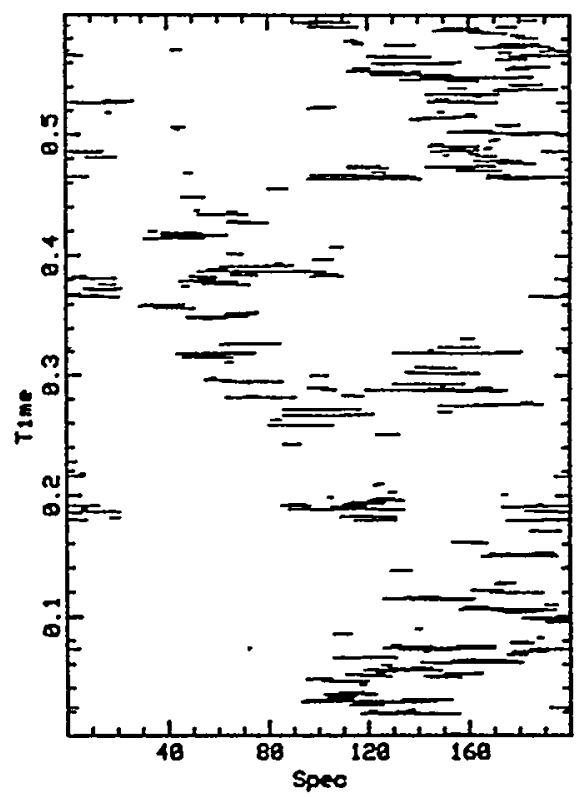

Fig. 11. Space-time plot of the activity. The horizontal axes is the "species axis." The time at which a given species mutates is shown as a black dot. The avalanches appear as connected black areas. On the time resolution of the plot, the avalanches appear as almost horizontal lines. Calculation was done for a value of the mutation parameter $T=0.01$.

During an avalanche, individual species may undergo many mutations, so their properties, or "morphologies," can change significantly, even if the effect of individual mutations is small. Thus, on a timescale that is large compared with that of the avalanche, evolution sometimes appears to take place in terms of saltations. There is a close connection between the punctuations of evolution (and the periods of stasis) of the individual species, and the global intensity of evolutionary activity. They are two sides of the same coin: the selforganized critical state. The punctuated equilibrium behavior of single species is a collective effect. The macroscopic jumps between "useful" or highly fit states are effectuated by the cumulative small jumps through intermediate states, which only could exist in the temporary environment of a burst.

For example, a grounded species may develop wings rapidly trough a sequence of smaller steps in a turbulent environment, where such development would be impossible in periods of stasis due to competition from other highly 
adapted species. Maybe this mechanism can soften Mivart's [30] critique of Darwin's theory for the "incompetency of natural selection to account for the incipient stages of useful structures."

Figure $8 \mathrm{~b}$ shows the accumulated number of mutations for a single species versus time' in the model. The number of mutations might be seen as a measure of the change in morphology for that particular species. The evolution shows punctuated equilibrium behavior. For more data see also Ref. [6] and references therein, e.g. the well documented Elephant lineages of Ref. [31].

If the evolutionary activity, as measured for instance in terms of the number of extinctions, is measured for an extended period, there will in general be several smaller and larger avalanches taking place during that period. One can prove mathematically that the distribution of activity in such intervals will converge towards a Pareto-Levy distribution function in the limit where there are many avalanches. This Pareto-Levy distribution has a power-law tail with an exponent equal to that of the individual avalanches. In Figure $6 \mathrm{~b}$, we have coarse-grained the time scale of an ecology of 128 species evolving over a total of $2^{14}$ steps, in 60 equal time intervals. The large fluctuations seen in the distribution of individual avalanches remain. The Pareto-Levy distribution is not a power law for small events. In order to extend the power-law downwards it is important to coarse grain over small intervals. Thus it would be nice to see the histogram of fossil extinctions measured on a finer time scale, say a million years.

If the value of the mutation parameter $T$ is increased, some regions of the ecology decorrelate, and the predicted power laws will be limited to a scaling regime which diminishes with increasing values of $T$. For this reason a detailed study of scaling behaviour for fossil records of subsets of the ecology may help to determine better the avalanche exponent. At higher values of the mutation parameter $T$, i.e. for higher mutation rates, well defined punctuations gradually cease to exist, and the evolutionary activity changes nature from intermittent to continuous. Well-defined periods of stasis disappear, and evolution as we know it with ecologies in apparent balance most of the time,

\section{Discussion and Conclusions}

For the present model to have any chance of representing evolution in Nature, it is important that those of its properties that we have focussed on here are robust and fundamentally unchanged by essentially any modification of the model that leave its defining elements unchanged. We have simulated many versions of the model with many different representations of the interactions between species. In all cases we found punctuated equilibrium with exponents depending only on the dimension of the lattice.

Our results demonstrate the advantage of simple "toy" models over more complicated, and supposedly more realistic, models. Not only are the toy 
models numerically tractable, but they are also exactly mathematically solvable in some respects. For the Random Neighbour Model described in [27] one can explicitly prove that it self-organizes to the critical state and find a number of properties of that state $[27,29]$. The mechanism for self-organized criticality in the general case of models with assigned neighbors has been identified by Paczuski et al. [28]. Having completed the present study, one could in principle return to the more elaborate representations of the landscape for the single species, as for instance the spin-glass model [25], or the NKC model [14]. Based on our observation of robustness, we conjecture that our conclusions, including the specific values of the exponents, will remain unaltered.

One important observation from this study is that Darwinian evolution acting on the level of the individual does not converge towards a state where every species is maximally fit, i.e. a state in which all barrier values $B$ are maximal. In a non-interactive ecology, this would eventually happen, but the time-scale would be enormous since one would have to wait for the occurrence of states with very high $B$-values, and therefore very low transition rates. The ecology discussed here evolves relatively fast to the globally correlated critical state, and once it has arrived there, it keeps evolving forever, alternating between periods of stasis and intermittent spikes of co-evolutionary activity of all sizes. According to this scenario, Life in its normal state is synonymous with volatility, not with stability and fitness. Darwins principle does not translate directly to the whole ecosystem, which in fact does not evolve towards higher fitness or stability. The critical state is not "a nice place to be," contrary to what Kauffman suggested. As the least fit species mutates to improve its fitness, other species find their fitnesses reduced and soon mutate, too, possibly triggering changes throughout the ecology, as we have seen. As the fitness of any species is no more durable than the state of the species with which it interacts, all species experience a "Red Queen" effect: they are forced to keep evolving towards higher fitness just to maintain their fitness.

Can any of this be studied in the laboratory? One possibility is to study the dynamics of a limited ecology of very simple species on the molecular level [32] in order to identify the interplay between local punctuations at the level of single species and the evolution of the ecology as a whole.

\section{Acknowledgments}

PB is supported by Brookhaven National Laboratory, the Division of Materials Science, U.S. Department of Energy under Contract No. DE-AC0276CH00016. HF is supported by the Danish Natural Science Research Council, Grant No. 11-0244-1. KS is supported by the Danish Natural Science Research Council, Grant No. 11-0608-1. PB and HF thank the Isaac Newton Institute for its hospitality. 


\section{References}

1 Raup, D. M. \& Sepkoski, J. J. Jr. (1982) Science 215, 1501-1503.

2 Raup, D. M. (1986). Science 231, 1528.

3 Raup, D. M. \& Boyanjian, G. E. (1988) Paleobiology 14, 109-125.

4 Sepkoski, J. J., Jr.-(1993) Paleobiology 19, 43-51.

5 Gould, S. J. \& Eldredge, N. (1977) Paleobiology 3, 114-151;

6 Gould, S. J. \& Eldredge, N. (1993) Nature 366, 223-227.

7 Darwin, C. (1859) The Origin of Species by Means of Natural Selection. 6th ed. John Murray, London, D' Appleton, London, 1910.

8 Hallam, A. (1986) Nature 319, 765-768.

9 Newell, N. D. (1952) J. of Paleontology 26, 371-385.

10 Vrba, E. S. (1985) Suid-Afrikaanse Tydskrif Wetens 81, 229-236.

11 Alvarez, L. W., Alvarez, F. A., \& Michel, H. V. (1980) Science 208, 1095-1108.

12 Bak, P., Tang, C., \& Wiesenfeld, K. (1987) Phys. Rev. Lett. 59, 381- 384; (1988) Phys. Rev. A 38, 364-374. Bak, P. \& Chen. K. (1991) Scientific American 264(1), 46-53. Bak. P. \& Paczuski, M. (1993) Physics World 6 (12), 39-43.

13 Bak, P., Chen, K., \& Creutz, M. (1989) Nature 342, 780-781.

14 Kauffman, S. A. \& Johnsen, S. J. (1991) J. Theo. Biology 149, 467-506.

15 Bak, P. (1993) "Self-Organized Criticality and Gaia" in Thinking about Biology, Stein and F.J. Varela) Addison-Wesley, $255-268$.

16 Flyvbjerg, H. \& Lautrup, B (1992) Ph, 255-268. jerg, H., \& Lautrup

17 Ray, T. S. (1992) in Artifial) Phys. Rev. A 46, 6724-6730. of Complexity, Proc. Vol. X Life II, Santa Fe Institute Studies in the Sciences City, CA, 371-408.

18 Adami C. (1994)

19 Kellogg, D. E. (1975) Prganized Criticality in Living Systems, Caltec Preprint.

Paleobiology 1, 359-370.

20 Wright, S. (1982) Evolution 36, 427-443.

21 Lande, R. (1985) Proc. Natl. Acad. Sci. USA 82, 7641-7645.

22 Newman, C. M., Cohen, J. E., \& Kipnis, C. (1985) Nature (London) 315

23 MacBean, I. T., McKenzie, J. A., \& Parsons, P. A. (1971) Theor. Appl. Genet. 24 Parsons, P. A. (1983) The Evolutionary Biology of Colonizing Species (Cam-

25 Anderson, P. W. (1985) in Emerging Synthesis in Science. Proc. of the Founding Workshop of the Santa Fe Institute. (ed. D. Pines).

26 Bak. P. \& Sneppen, K. (1993) Phys. Rev. Lett. 71, 4083-4086.

27 Flyvbjerg, H., Sneppen, K. \& Bak, P. (1993) Phys. Rev. Lett. 71, 4087-4090.

28 Paczuski, M., Maslov, S., \& Bak, P: (1994), Europhys. Lett. in press.

29 Harris, T. E. (1963) The Theory of Branching Processes, (Springer, Berlin). submitted to Phys. Rev. Lett.

31 Mivart, S. G. J. (1871), On the Genesis of Species (D. Appleton \& Co., New York).

32 Maglio, V.J. (1973) Amer. Philos. Soc. Trans. 63, 1-149; For an excellent survey of evolution diagrams and macroevolution in general, see Stanley, S.M. (1979)
Macroevolution, Patterns and Process (W.H. Freeman and Co., San Francisco).

33 Eigen, M. \& Schuster, P. (1993) The Hypercycle. (Springer-Verlag, Berlin, Hei-

34 Eigen, M. \& Schuster, P. (1977) Naturwissenschaften 65, 541; (1978) idem 65, 